\title{
A THEORY OF COVERING AND DIFFERENTIATION
}

\author{
BY \\ ANTHONY P. MORSE
}

Contents

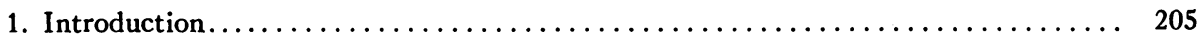

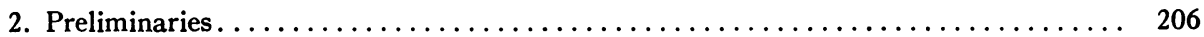

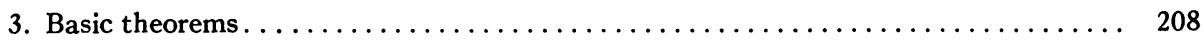

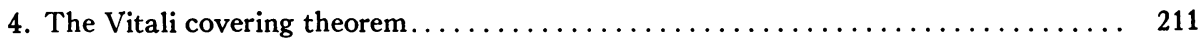

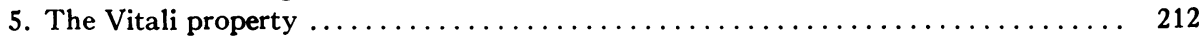

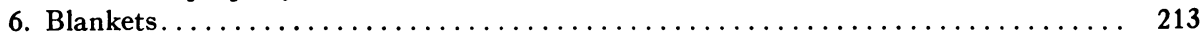

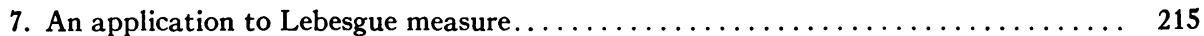

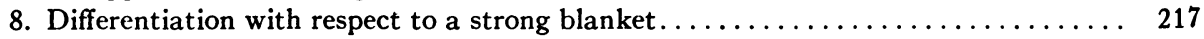

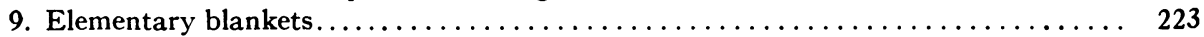

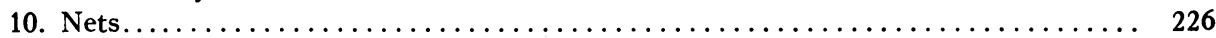

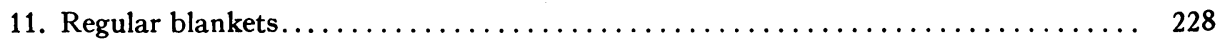

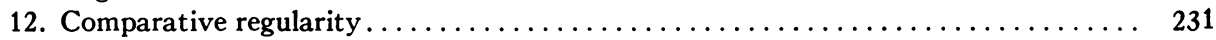

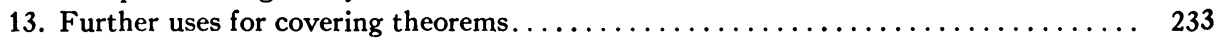

1. Introduction. The isolation in 3.10 of a certain basic principle makes possible a covering theory which not only embraces every known $\left({ }^{1}\right)$ theorem of the Vitali type but enables us to supplant the usual $\left({ }^{2}\right)$ abstract differential theory with one more in accord with Lebesgue's. In fact, by substituting a new and frequently useful concept of regularity for the classical notion $\left({ }^{3}\right)$, we arrive at a general theory of differentiation which both includes and supplements similar results of Lebesgue.

We follow Banach's lead $\left(^{4}\right)$ in paying special attention to nonadditive functions. By so doing a rather striking difference appears between the differential theory of interval functions on the one hand and that of set functions on the other.

As could be gathered from $\S 2$, we shall be concerned with a metric ( $\left.{ }^{5}\right)$ space $\mathcal{S}$, a fixed measure $\phi$, and certain other measures. We presuppose such a knowledge of measure as might be derived from $\S 3$ of the subjoined paper $\left(^{6}\right)$ by Randolph and myself. Although this $\$ 3$ of RM concerns itself with cer-

Presented to the Society, October 30,1943; received by the editors March 2, 1943, and, in revised form, September 4, 1943.

(1) See J. F. Randolph, The Vitali covering theorem for Caratheodory linear measure, Ann. of Math. vol. 40 (1939) pp. 299-308; S. Saks, Theory of the integral, Warsaw, 1937, pp. 106-112; and related papers referred to by Randolph and Saks.

(2) Saks, loc. cit. pp. 152-156.

(3) Saks, loc. cit. p. 106.

(4) S. Banach, Sur une classe de fonctions d'ensemble, Fund. Math. vol. 6 (1924) pp. 170-188.

(b) Except for applications the metric plays a minor role; it is easy to interpret the paper topologically.

(6) Hereinafter referred to as RM. 
tain plane measures the reader should easily see that the results therein obtained hold equally well (with the obvious interpretations) when the plane is replaced by an abstract metric space. So extended, Theorems 3.7 and 3.13 of $R M\left({ }^{7}\right)$ are used in the present paper $(\mathrm{M})$. On the other hand Theorem 11.4 of $\mathrm{M}$ is used in proving Theorem 10.1 of $\mathrm{RM}$. This cross reference is noncircular since $\S 3$ of $R M$ is entirely independent of $M$.

2. Preliminaries. The phrase " $x \in A$ " is read " $x$ is a member of $A$ " or more simply " $x$ is in $A$." The phrase " $x \notin A$ " is read " $x$ is not a member of $A$ " or more simply " $x$ is not in $A$." The phrase " $A \subset B$ " is read " $A$ is contained in $B$ " or " $A$ is a subset of $B$. " Reversely the phrase " $B \supset A$ " is read " $B$ contains $A$."

The integer 0 and the vacuous set will be regarded as equal.

We employ $\{x\}$ as short for

$$
\underset{y}{E}[x=y]
$$

and use \{\} only in this sense. Note that $x$ is the sole member of $\{x\}$.

If $a_{x}$ is a set for each $x$ in a set $B$, then

$$
\sum_{x \in B} a_{x}
$$

is defined as

$$
\underset{y}{E}\left[y \in a_{x} \text { for some } x \text { in } B\right] \text {. }
$$

If $\mathfrak{F}$ is a family (set), then $\sigma(\mathfrak{F})$ is defined as

$$
\sum_{\beta \in \mathcal{F}} \beta \text {. }
$$

Thus $x \in \sigma(\mathfrak{F})$ if and only if $x \in \beta$ for some $\beta$ in $\mathfrak{F}$.

If $a_{x} \geqq 0$ for $x$ in a countable set $B$, then the notation

$$
\sum_{x \in B} a_{x}
$$

will also be used to denote the appropriate numerical sum. Whether numerical summation or set theoretic summation is intended in a given instance will be obvious from the context.

A family $B$ will be called disjointed if and only if $B_{1} B_{2}=0$ whenever $B_{1}$ and $B_{2}$ are different members of $B$.

We fix throughout the paper a space $S$ metrized with $\rho$. More specifically we assume henceforth that $\rho$ is such a function on $E_{(x, y)}[(x \in \mathcal{S})(y \in S)]$ that

$$
\rho(x, x)=0 \leqq \rho(x, z) \leqq \rho(x, y)+\rho(z, y)<\infty
$$

whenever $x, y$, and $z$ are in $S$. It follows that $\rho(x, y)=\rho(y, x)$ for $x$ and $y$

( 7 These general results are apparently unknown and unpublished elsewhere. 
in $S$, but it does not follow nor do we assume (though the reader may wish to) that $x=y$ whenever $\rho(x, y)=0$. Accordingly a finite subset of $S$ may fail to be closed.

We also fix throughout the paper a measure $\phi$ belonging to the class $\mathfrak{i}$ which is defined in 2.3 below.

2.1. Definition. The family $\mathfrak{T}$ is defined by: $\psi \in \mathfrak{T}$ if and only if $\psi$ is such a function on $E_{S}[S \subset S]$ to $E_{t}[0 \leqq t \leqq \infty]$ that:

I. $\psi(0)=0$;

II. $\psi(B) \leqq \psi(A)$ whenever $B \subset A \subset S$;

III. If $\mathfrak{F}$ is a countable family with $\sigma(\mathfrak{F}) \subset \mathcal{S}$, then

$$
\psi[\sigma(\mathfrak{F})] \leqq \sum_{S \in \mathfrak{F}} \psi(S) \text {. }
$$

2.2. Definition. The family $\mathfrak{U}$ is defined by: $\psi \in \mathfrak{U}$ if and only if $\psi$ is such a member of $\mathfrak{T}$ that

IV. $\psi(A+B)=\psi(A)+\psi(B)$ whenever

$$
\inf _{x \in A, y \in B} \rho(x, y)>0 .
$$

2.3. Definition. We define

$$
\ddot{\mathfrak{U}}=\mathfrak{u} \underset{\psi}{E}[\psi(S)<\infty \text { for each bounded set } S] .
$$

Due to Carathéodory is the following

2.4. Definition. We say $A$ is $\psi$ measurable if and only if $\psi$ is such a member of $\mathfrak{T}$ that

$$
\psi(T)=\psi(T A)+\psi(T S-T A)
$$

for each subset $T$ of $S$.

2.5. Remark. Clearly $\mathfrak{i} \subset \mathfrak{u}$.

Information concerning measures in $\mathfrak{U}$ can be found in $\$ 3$ of RM. See also pp. 424-444 of H. Hahn, Theorie der reellen Funktionen. I, Berlin, 1921.

Hereafter such words as measurable, measure zero, almost all, and so on, will refer to the fixed measure $\phi$ belonging to $\mathfrak{i}$ just as such words as closed, open, Borel, and so on, refer to the metric $\rho$ metrizing $S$.

2.6. Definition. For $A \subset S$ we define:

$$
\begin{array}{ll}
\operatorname{diam} A=\sup _{x \in A, \nu \in A} \rho(x, y) & \text { whenever } A \neq 0 ; \\
\operatorname{diam} A=0 & \text { whenever } A=0 .
\end{array}
$$

2.7. Definition. For $A \subset S$ we define

$$
\hat{A}=\underset{x}{E}\left[\rho(x, y) \leqq 2^{\circ} \operatorname{diam} A \text { for some } y \text { in } A\right] .
$$

2.8. Definition. If $\Delta$ is a non-negative function whose domain is a family 
$\mathfrak{S}$, then corresponding to each $\beta$ in $\mathfrak{S}$ we define $\Delta: \beta$ as the set $S$ for which $x \in S$ if and only if there is such a set $\alpha$ in $\mathfrak{W}$ that

$$
x \in \alpha, \quad \beta \alpha \neq 0, \quad \Delta(\alpha) \leqq 2 \Delta(\beta) .
$$

2.9. Definition. The limit notations

$$
\varliminf_{\mathfrak{i} \ni \beta \rightarrow x} f(\beta), \quad \varlimsup_{\mathfrak{F} \ni \beta \rightarrow x} f(\beta),
$$

in which $\mathfrak{F}$ is a family of subsets of $\mathcal{S}$ and $x \in \mathcal{S}$, are defined respectively as

$$
\lim _{t \rightarrow 0+} \inf _{\beta \in H(t)} f(\beta), \quad \lim _{t \rightarrow 0+} \sup _{\beta \in H(t)} f(\beta)
$$

where

$$
H(t)=. \mathfrak{F} \underset{\beta}{E}[\operatorname{diam}(\{x\}+\beta)<t]
$$

for $t>0$.

We agree that

$$
\operatorname{limal}_{\mathfrak{i} \ni \beta \rightarrow x} f(\beta)=\varlimsup_{\mathfrak{i} \ni \beta \rightarrow x} f(\beta)
$$

if and only if

$$
\varlimsup_{\mathfrak{i} \ni \beta \rightarrow x} f(\beta)=\operatorname{limal}_{\mathfrak{F} \ni \beta \rightarrow x} f(\beta) .
$$

It may clarify our intent to point out that

$$
\operatorname{limal}_{\mathcal{F} \ni \beta \rightarrow x} f(\beta)=f(\{x\})
$$

whenever the left member denotes a number and $\{x\} \in \mathfrak{F}$.

\section{Basic theorems.}

3.1. Definition. We say $N$ is a nest whenever $N$ is such a family that if $\mathfrak{W}_{1} \in N$ and $\mathfrak{W}_{2} \in N$ then either $\mathfrak{W}_{1} \subset \mathfrak{W}_{2}$ or $\mathfrak{W}_{2} \subset \mathfrak{W}_{1}$.

Of use to us is the following

3.2. Lemma. If $\Omega$ is a family of sets then $\left(^{8}\right) \Omega$ contains such a nest $N$ that no member of $\Omega-N$ contains every member of $N$.

3.3. THEOREM. If $\mathfrak{F}$ is a family of nonvacuous subsets of $\mathcal{S}$, and $\Delta$ is a nonnegative function which is bounded on $\mathfrak{F}$, then $\mathfrak{F}$ contains such a disjointed family (B) that each $\beta$ in $F$ intersects some $B$ in \& in such a way that $\beta C \Delta: B$.

(8) This lemma is an immediate consequence of a result due to C. Kuratowski, Une méthode d'élimination des nombres transfinis des raisonnements mathematiques, Fund. Math. vol. 3 (1922) p. 89, formulation (42). See also M. Zorn, Bull. Amer. Math. Soc. vol. 41 (1935) p. 667; and J. W. Tukey, Convergence and uniformity in topology, Princeton, 1940, p. 7. 
Proof. For $\mathfrak{W \subset F ~ l e t ~}$

$$
\mathfrak{S}^{+}=\mathfrak{F} \underset{\beta}{E}[\beta \sigma(\mathfrak{W}) \neq 0]
$$

Let $\Omega$ be the family for which $\mathfrak{T} \in \Omega$ if and only if $\mathfrak{E}$ is such a disjointed subfamily of $\mathfrak{F}$ that each $\beta$ in $\mathfrak{S}^{+}$intersects some $B$ in $\mathfrak{W}$ in such a way that $\beta C \Delta: B$. Using 3.2 select such a nest $N$ that no member of $\Omega-N$ contains every member of $N$. Let $B=\sigma(N)$.

Since $N$ is a nest and since

$$
\text { (S) }=\sum_{\mathfrak{S} \in N} \mathfrak{S} \subset \mathfrak{F}, \quad \mathfrak{S}^{+}=\sum_{\mathfrak{S} \in N} \mathfrak{S}^{+},
$$

it is clear that $\mathscr{B}$ is such a disjointed subfamily of $\mathfrak{F}$ that each $\beta \in \mathcal{B}^{+}$intersects some $B$ in $B$ in such a way that $\beta \subset \Delta: B$.

Thus completion of the proof hinges on the

Statement. $\mathfrak{S}^{+}=\mathfrak{F}$.

Proof (By contradiction). Suppose $\mathfrak{S}^{+} \neq \neq \mathfrak{F}$, let $\Re=\mathfrak{F}_{-}-\mathfrak{S}^{+}$, note that $\Re \neq 0$, and invoke the boundedness of $\Delta$ to ascertain $B_{0} \in \Re$ so that

$$
\Delta(\beta) \leqq 2 \Delta\left(B_{0}\right) \text { for } \beta \in \Re .
$$

Inasmuch as $\oiint+\left\{B_{0}\right\}$ is evidently a member of $\Omega$ which contains every member of $N$ we infer

$$
\begin{aligned}
& \mathbb{S}+\left\{B_{0}\right\} \in N, \quad \mathbb{B}+\left\{B_{0}\right\} \subset \sigma(N)=\mathbb{S}, \quad B_{0} \in S^{*}, \\
& B_{0} \subset \sigma(\mathbb{S}), \quad 0 \neq B_{0}=B_{0} \sigma(\mathbb{S}), \quad B_{0} \in S^{+}, \quad B_{0} \notin S^{+} .
\end{aligned}
$$

3.4. THEOREM. If $A \subset \sigma(\mathfrak{F}) \subset S$ and $\Delta$ is a non-negative function which is bounded on $\mathfrak{F}$, then $\mathfrak{F}$ contains a disjointed family (S) for which

$$
A \subset \sum_{B \in \Theta} \Delta: B \text {. }
$$

Proof. Let $\mathfrak{F}^{\prime}=\mathfrak{F}-\{0\}$ and use 3.3 to obtain such a disjointed subfamily (S) of $\mathfrak{F}^{\prime}$ that

$$
A \subset \sigma(\mathfrak{F})=\sigma\left(\mathfrak{F}^{\prime}\right)=\sum_{\beta \in \mathfrak{F}^{\prime}} \beta \subset \sum_{B \in \circlearrowleft} \Delta: B .
$$

3.5. TheOREM. If $A \subset \sigma(\mathfrak{F}) \subset \mathcal{S}$ and

$$
\sup _{\beta \in \mathcal{F}} \operatorname{diam} \beta<\infty,
$$

then $\mathfrak{F}$ contains a disjointed family \& for which

$$
A \subset \sum_{B \in \leftrightarrow} \hat{B} \text {. }
$$

Proof. Let $\Delta$ be the function on $\mathfrak{F}$ such that 


$$
\Delta(\beta)=\operatorname{diam} \beta
$$

Use 3.4 to obtain such a disjointed subfamily $\mathbb{S}$ of $\mathfrak{F}$ that

$$
A \subset \sum_{B \in \Theta} \Delta: B \subset \sum_{B \in \Theta} \hat{B} \text {. }
$$

3.6. REMARK. In the applications of 3.4 and 3.5 it is frequently possible to infer the countability of $\&$ from its disjointedness.

3.7. Definition. We say $\mathfrak{F}$ covers $A$ if and only if $A \subset \sigma(\mathfrak{F})$.

3.8. Definition. We say $\mathfrak{F}$ covers almost all of $A$ if and only if

$$
\phi[A-\sigma(\mathfrak{F})]=0 .
$$

3.9. Definition. We say $\mathfrak{F}$ covers $A$ in the sense of Vitali if and only if $\mathfrak{F}$ is such a family of closed subsets of $S$ that corresponding to each positive number $\epsilon$ and each $x \in A$ there is a set $\beta \in \mathfrak{F}$ for which $x \in \beta$ and $\operatorname{diam} \beta<\epsilon$.

3.10. THEOREM. If $\mathfrak{F}$ covers $A$ in the sense of Vitali and $\Delta$ is a non-negative function which is bounded on $\mathfrak{F}$, then $\mathfrak{F}$ contains a disjointed family $\$$ for which

$$
A-\sigma(\mathfrak{S}) \subset \sum_{B \in \mathfrak{S}} \Delta: B
$$

whenever $\mathfrak{S C B}$ and $\mathfrak{B}-\mathfrak{S}$ is finite.

Proof. Let

$$
\mathfrak{F}^{\prime}=\mathfrak{F}-\{0\}
$$

and note that $\mathfrak{F}^{\prime}$ likewise covers $A$ in the sense of Vitali. In accordance with 3.3 select such a disjointed subfamily $\mathbb{F}$ of $\mathfrak{F}^{\prime}$ that each $\beta \in \mathfrak{F}^{\prime}$ intersects some $B$ in $B$ in such a way that $\beta \subset \Delta: B$.

We suppose

$$
\mathfrak{S C G}, \quad \mathfrak{S}^{\prime}=\mathfrak{S}-\mathfrak{S}, \quad \mathfrak{Q}^{\prime} \text { is finite, }
$$

and complete the proof of the theorem with the verification of the

Statement. If $x \in A-\sigma(\mathfrak{S})$ then $x \in \sum_{B \in \mathfrak{\$}} \Delta: B$.

Proof. Inasmuch as $\mathfrak{S}^{\prime}$ is a finite family of closed sets it follows that $\sigma\left(\mathfrak{Q}^{\prime}\right)$ is closed. From

$$
x \in A-\sigma(\mathfrak{S}) \subset A-\sigma\left(\mathfrak{S}^{\prime}\right)
$$

we infer $x \notin \sigma\left(\mathfrak{S}^{\prime}\right)$ and use the closedness of $\sigma\left(\mathfrak{S}^{\prime}\right)$ and the fact that $\mathfrak{F}^{\prime}$ covers $\{x\}$ in the sense of Vitali to secure such a set $\beta_{0} \in \mathscr{F}^{\prime}$ that $x \in \beta_{0}$ with $\beta_{0} \sigma\left(\mathfrak{S}^{\prime}\right)=0$. Our selection of $\mathbb{B}$ enables us to ascertain $B_{0}$ in $\mathbb{B}$ so that

$$
\beta_{0} B_{0} \neq 0, \quad \beta_{0} \subset \Delta: B_{0}
$$

and our choice of $\beta_{0}$ thus allows us to conclude 


$$
B_{0} \notin \mathfrak{S}^{\prime}, \quad B_{0} \in \mathfrak{S}, \quad x \in \beta_{0} \subset \Delta: B_{0} \subset \sum_{B \in \mathfrak{W}} \Delta: B .
$$

4. The Vitali covering theorem. The first of our theorems to involve the measure $\phi$, which was fixed in $\S 2$, is

4.1. The Vitali covering theorem. If

(i) $0<\lambda<\infty$,

(ii) $A$ is a bounded subset of $\mathrm{S}$,

(iii) $\Delta$ is a non-negative function which is bounded on $\mathfrak{F}$,

(iv) $\mathfrak{F}$ so covers $A$ in the sense of Vitali that

$$
\phi(\Delta: \beta)<\lambda \phi(\beta)
$$

for $\beta \in \mathfrak{F}$

then $\mathfrak{F}$ contains a countable disjointed family which covers almost all of $A$.

Proof. Take a bounded open set $S$ which contains $A$, let

$$
\mathfrak{F}^{\prime}=\mathfrak{F} \underset{\beta}{E}[\beta \subset S]
$$

note that $\mathfrak{F}^{\prime}$ covers $A$ in the sense of Vitali, use 3.10 to select such a disjointed family $\&$ of $\mathfrak{F}^{\prime}$ that

$$
A-\sigma(\mathfrak{S}) \subset \sum_{B \in \mathfrak{S}} \Delta: B
$$

whenever $\mathfrak{E} \subset \mathbb{S}$ and $\mathbb{S}-\mathfrak{S}$ is finite.

Since (iv) guarantees

$$
\phi(\beta)>0
$$

for $\beta \in \mathfrak{F}$,

the countability of $B$ follows from the boundedness of $A$, the disjointed nature of $\mathbb{B}$, and the membership of $\phi$ in the (2.3) family $\mathfrak{i}$.

We complete the proof with the verification of the

Statement. $\phi[A-\sigma(\$)]=0$.

Proof. Let $\epsilon>0$. After checking

$$
\sum_{B \in \Theta} \phi(\Delta: B) \leqq \lambda \sum_{B \in \Theta} \phi(B)=\lambda \phi[\sigma(B)] \leqq \lambda \phi(S)<\infty
$$

we select from $\mathbb{B}$ a subfamily $\mathfrak{Q}$ for which $\mathbb{S}-\mathfrak{S}$ is finite,

$$
\sum_{B \in \mathfrak{S}} \phi(\Delta: B)<\epsilon .
$$

In the light of (1) we see

$$
\phi[A-\sigma(\mathfrak{S})] \leqq \sum_{B \in \mathfrak{\Phi}} \phi(\Delta: B)<\epsilon .
$$

4.2. REMARK. The validity of 4.1 is unchanged by the additional assump- 
tion therein of the countability of $\mathfrak{F}$ and the replacement, in Definition 2.8, of the factor " 2 " by " 1 ". This modified version of 4.1 is adequate for many purposes and is easily proved by straightforward considerations of finite families.

\section{The Vitali property.}

5.1. Definition. We say $\mathfrak{F}$ has the Vitali property with respect to $A$ whenever $\mathfrak{F}$ is such a family of measurable sets that corresponding to each finite disjointed subfamily $\mathfrak{S}$ of $\mathfrak{F}$ there is a countable disjointed family (B) for which

$$
\mathfrak{S} \subset \mathfrak{S} \subset \mathfrak{F}, \quad \phi[A-\sigma(\mathfrak{S})]=0 .
$$

5.2. THEOREM. If $\Omega$ is a nonvacuous countable family of sets with respect to each of which $\mathfrak{F}$ has the Vitali property, then $\mathfrak{F}$ has the Vitali property with respect to $\sigma(\Omega)$.

Proof. Let $\mathfrak{S}$ be a finite disjointed subfamily of $\mathfrak{F}$. Let $A=\sigma(\Omega)$. Let $\Lambda$ be such a repetitive sequence of members of $\Omega$ that

$$
A=\sum_{j=n}^{\infty} \Lambda_{j}
$$

for each positive integer $n$. Let $S$ be such a sequence of bounded open sets that

$$
S_{1} \subset S_{2} \subset S_{3} \subset \cdots, \quad \delta=S_{1}+S_{2}+S_{3}+\cdots \text {. }
$$

Next use the theorem's hypotheses and the fact that $\phi\left(S_{n}\right)<\infty$ for each positive integer $n$ to ascertain such a sequence $G$ of finite disjointed families that

$$
\mathfrak{S} \subset G_{j} \subset G_{j+1} \subset \mathfrak{F}, \quad \phi\left[S_{j} \Lambda_{j}-\sigma\left(G_{j}\right)\right] \leqq 1 / 2^{j}
$$

for each positive integer $j$. Finally let

$$
\text { (S) }=\sum_{j=1}^{\infty} G_{j} .
$$

It is clear that $B$ is a countable disjointed family for which $\mathfrak{S} \subset \mathscr{S} \subset \mathfrak{F}$. Moreover for each positive integer $n$ we have:

$$
\begin{aligned}
A & =\sum_{j=n}^{\infty} S_{j} \Lambda_{j} ; \\
\phi[A-\sigma(\mathcal{S})] & \leqq \sum_{j=n}^{\infty} \phi\left[S_{j} \Lambda_{j}-\sigma(\mathcal{S})\right] \\
& \leqq \sum_{j=n}^{\infty} \phi\left[S_{j} \Lambda_{j}-\sigma\left(G_{j}\right)\right] \leqq \sum_{j=n}^{\infty} \frac{1}{2^{j}}=\frac{1}{2^{n-1}} .
\end{aligned}
$$

Accordingly $\phi[A-\sigma(\mathcal{S})]=0$ and $\mathfrak{F}$ has the Vitali property with respect to $A$. The proof is complete. 
6. Blankets. In order to discuss more intricate covering theorems and facilitate a theory of differentiation we introduce the notion of blanket.

6.1. Definition. We say $F$ is a blanket if and only if $F$ is such a function that for $x$ in its domain:

(i) $x \in \mathcal{S}$ and $F(x)$ is a family of nonvacuous subsets of $S$;

(ii) $\beta \in F(x)$ implies diam $\beta<\infty$;

(iii) $\inf _{\beta \in F(x)} \operatorname{diam}(\beta+\{x\})=0$.

Evidently the domain of a blanket is a subset of $S$.

In 6.2 through 6.10 we define certain words used in connection with blankets. These words are: spread, natural, regular, diametrically regular, Borelish, close, heavy, subblanket, strong.

6.2. Definition. If $F$ is a blanket with domain $A$ then the set

$$
\sum_{x \in A} F(x)
$$

is called the spread of $F$.

6.3. Definition. We say $F$ is a natural blanket whenever $F$ is such a blanket that

$$
\beta \in F(x) \text { implies } \quad x \in \beta .
$$

Definition 2.9 is used in

6.4. Definition. If $F$ is natural blanket and if there is such a non-negative function $\Delta$ that

$$
\varlimsup_{F(x) \ni \beta \rightarrow x}\left[\Delta(\beta)+\frac{\phi(\Delta: \beta)}{\phi(\beta)}\right]<\infty
$$

for almost all $x$ in the domain of $F$, then $F$ is a regular blanket.

6.5. Definition. We say $F$ is a diametrically regular blanket whenever $F$ is such a natural blanket that

$$
\varlimsup_{F(x) \ni \beta \rightarrow x} \frac{\phi(\hat{\beta})}{\phi(\beta)}<\infty
$$

for almost all $x$ in the domain of $F$.

Evidently every diametrically regular blanket is regular.

Many of the blankets met with in practice are diametrically regular.

6.6. Definition. We say $F$ is a Borelish blanket whenever $F$ is such a blanket that

$$
\beta \in F(x) \text { implies } \beta \text { is a Borel set. }
$$

6.7. Definition. We say $F$ is a close blanket whenever $F$ is such a blanket that 


$$
\beta \in F(x) \text { implies } \beta \text { is a closed set. }
$$

6.8. Definition. If $F$ is a Borelish blanket, with domain $A$, and if the spread of $F$ contains a countable disjointed family which covers almost all of $A$, then $F$ will be called a heavy blanket.

6.9. Definition. We say $G$ is a subblanket of $F$ whenever $G$ and $F$ are such blankets that $G(x) \subset F(x)$ for $x$ in the domain of $G$.

Clearly the domain of $G$ is contained in the domain of $F$ and the spread of $G$ is contained in the spread of $F$ whenever $G$ is a subblanket of $F$.

6.10. Definition. If $F$ is such a blanket that each of its subblankets is a heavy blanket then $F$ will be termed a strong blanket.

\subsection{LEMMA. Every regular close blanket is a heavy blanket.}

Proof. Let $F$ be any regular, close blanket with domain $A$, denote the spread of $F$ by $\mathfrak{F}$, and choose such a non-negative function $\Delta$ that

$$
\varlimsup_{\boldsymbol{F}(x) \ni \beta \rightarrow x}\left[\Delta(\beta)+\frac{\phi(\Delta: \beta)}{\phi(\beta)}\right]<\infty
$$

for almost all $x$ in $A$. Select bounded sets $S_{1} \subset S_{2} \subset S_{3} \subset \cdots$ so that

$$
S=S_{1}+S_{2}+S_{3}+\cdots
$$

and define

$$
A_{n}=S_{n} \underset{x}{E}\left[\varlimsup_{F(x) \ni \beta \rightarrow x}\left(\Delta(\beta)+\frac{\phi(\Delta: \beta)}{\phi(\beta)}\right)<n\right] .
$$

Let

$$
\Re=\sum_{n=1}^{\infty}\left\{A_{n}\right\}
$$

and note

$$
\phi[A-\sigma(\Re)]=0 .
$$

The remainder of the proof consists of three parts.

PART I. If $n$ is a positive integer then $\mathfrak{F}$ has the Vitali property with respect to $A_{n}$.

Proof. Let $\mathfrak{S}$ be any finite disjointed subfamily of $\mathfrak{F}$. Let $B=A_{n}-\sigma(\mathfrak{Q})$ and

$$
\mathfrak{F}^{\prime}=\mathfrak{F} \underset{\beta}{E}[\beta \sigma(\mathfrak{W})=0] \underset{\beta}{E}\left[\Delta(\beta)+\frac{\phi(\Delta: \beta)}{\phi(\beta)}<n\right] .
$$

From (1) and the closedness of $\sigma(\mathfrak{S})$ we gather that $\mathfrak{F}^{\prime}$ covers $B$ in the sense of Vitali. Thus with the help of 4.1 we secure such a countable disjointed subfamily $\mathcal{B}^{\prime}$ of $\mathfrak{F}^{\prime}$ that 


$$
\phi\left[B-\sigma\left(\mathbb{S}^{\prime}\right)\right]=0 .
$$

Letting $\mathfrak{B}^{\prime}=\mathbb{S}^{\prime}+\mathfrak{S}$ and using the definition of $\mathfrak{F}^{\prime}$ we infer

$$
\sigma\left(\mathfrak{S}^{\prime}\right) \sigma(\mathfrak{E}) \subset \sigma\left(\mathfrak{F}^{\prime}\right) \sigma(\mathfrak{W})=0
$$

and deduce that $\mathscr{S}$ is a countable disjointed family for which $\mathfrak{E} \subset \mathscr{S} \subset \mathfrak{F}$. We also have

$$
\begin{aligned}
\phi\left[A_{n}-\sigma(\mathfrak{S})\right] & \leqq \phi[\sigma(\mathfrak{S})-\sigma(\mathbb{S})]+\phi\left[B-\sigma\left(\$^{\prime}\right)\right] \\
& \leqq 0+\phi\left[B-\sigma\left(\mathbb{S}^{\prime}\right)\right]=0+0=0 .
\end{aligned}
$$

PART II. F has the Vitali property with respect to $A$.

Proof. Use (2), (3), Part I, 5.2.

PART III. $\mathfrak{F}$ is a heavy blanket.

Proof. Use Part II, take $\mathfrak{E}=0$ in 5.1 , recall Definition 6.8 of a heavy blanket.

6.12. TheOREM. Every regular close blanket is a strong bla nket.

Proof. Let $F$ be a regular close blanket. Let $F^{\prime}$ be any subblanket of $F$. Since $F^{\prime}$ is manifestly a regular close blanket we conclude from 6.11 that it is heavy.

Reference to 6.10 completes the proof.

7. An application to Lebesgue measure. In this section we suppose $n$ is a positive integer, $S$ is $n$-space, $\rho$ is the usual metric, $\phi$ is $n$-dimensional Lebesgue measure.

7.1. Definition. We say $\mathfrak{E}$ is a hive whenever $\mathfrak{E}$ is such a family of bounded closed convex subsets of $S$ that:

(i) $\beta \in \mathfrak{S}$ implies $\phi(\beta)>0$;

(ii) $\left(\beta_{1} \in \mathfrak{W}\right)\left(\beta_{2} \in \mathfrak{W}\right)$ implies $\left(\beta_{1}\right.$ either contains or is contained in some translate of $\beta_{2}$ ).

7.2. ThEOREM. If $F$ is a natural blanket whose spread is a hive then $F$ is a regular close blanket.

Proof. Let $\mathfrak{F}$ be the spread of $F$. Let $q$ be such a positive integer that

$$
2<\left(1+1 / 2^{n}\right)^{q} \text {. }
$$

Let $\Delta$ be the function on $\mathfrak{F}$ such that $\beta \in \mathfrak{F}$ implies

$$
\Delta(\beta)=[\phi(\beta)]^{q} .
$$

Corresponding to each convex subset $\beta$ of $\mathcal{S}$ let us define $\beta^{\nabla}$ as the set of points of the form $2 x-y$ where $x$ and $y$ are members of $\beta$. Whenever $\beta$ is a convex subset of $S$ it is evident that $\beta^{\nabla}$ is convex and is equal to the set of points of the form $x+(y-z)$ where $x, y$, and $z$ are members of $\beta$.

We divide the remainder of the proof into two parts. 
PART I. If $\alpha$ and $\beta$ are such members of $\mathfrak{F}$ that

$$
\beta \alpha \neq 0, \quad \Delta(\alpha) \leqq 2 \Delta(\beta)
$$

then

$$
\alpha \subset \beta^{\nabla \nabla} \text {. }
$$

Proof. If some translate of $\beta$ contains $\alpha$ then we clearly have

$$
\alpha \subset \beta^{\nabla} \subset \beta^{\nabla \nabla} \text {. }
$$

We now assume that some translate of $\alpha$ contains $\beta$ and let $\alpha_{0}$ be such a translate of $\alpha$.

In order to show $\alpha_{0} \subset \beta^{\nabla}$ we suppose the contrary; let $x_{0}$ be a point in $\alpha_{0}-\beta^{\nabla}$; define $\beta^{\prime}$ as the set of points of the form

$$
\left(x_{0}+y\right) / 2
$$

where $y$ is a member of $\beta$; verify that $\beta \beta^{\prime}=0, \beta+\beta^{\prime} \subset \alpha_{0}$ and that

$$
\left(1+1 / 2^{n}\right) \phi(\beta)=\phi(\beta)+\phi\left(\beta^{\prime}\right)=\phi\left(\beta+\beta^{\prime}\right) \leqq \phi\left(\alpha_{0}\right)=\phi(\alpha) ;
$$

arrive at the contradiction

$$
2 \Delta(\beta)=2[\phi(\beta)]^{q}<\left[\left(1+1 / 2^{n}\right) \phi(\beta)\right]^{q} \leqq\left[\phi(\alpha)^{q}\right]=\Delta(\alpha) .
$$

Since we now know $\alpha_{0} \subset \beta^{\nabla}$ we are able to conclude

$$
\alpha \subset \alpha_{0}^{\nabla} \subset \beta^{\nabla \nabla} .
$$

PART II. If $\beta \in F$ then

$$
\phi(\Delta: \beta) \leqq(n+2)^{2 n} \phi(\beta) .
$$

Proof. From Part I we deduce

$$
\Delta: \beta \subset \beta^{\nabla \nabla} \text {. }
$$

If $\beta$ has central symmetry, it is obvious that

$$
\phi\left(\beta^{\nabla}\right)=3^{n} \phi(\beta), \quad \phi\left(\beta^{\nabla \nabla}\right)=3^{2 n} \phi(\beta) .
$$

However even if $\beta$ doesn't have central symmetry it is possible $\left(^{(}\right)$to show

$$
\phi\left(\beta^{\nabla}\right) \leqq(n+2)^{n} \phi(\beta), \quad \phi\left(\beta^{\nabla \nabla}\right) \leqq(n+2)^{2 n} \phi(\beta) .
$$

PART III. $F$ is a regular close blanket.

Proof. Use Part II.

7.3. REMARK. Theorem 7.2 includes the covering theorem alluded to by B. Jessen, J. Marcinkiewicz, and A. Zygmund on page 224 of Fund. Math.

( $\left.{ }^{9}\right)$ Use T. Bonnesen and W. Fenchel, Theorie der konvexen Körper, Ergebnisse der Mathematik vol. 3 (1934) p. 53, formula (1). 
vol. 25 (1935). In view of Theorem A of that paper it is of interest that a satisfactory differential theory holds for a large class of rectangles which are allowed to be long, thin, and oriented in every direction.

Because of our 6.12 it follows that Theorem 6 of the paper just cited is a consequence of Theorem 8.12 below.

7.4. REMARK. Although some changes in proof would be required, the validity of 7.2 would be unimpaired by the introduction into 7.1 (ii) of certain homothetical transformations.

8. Differentiation with respect to a strong blanket. In canvassing the possible applications of the present section it is well to bear Theorem 6.12 in mind.

The next three definitions play a central role in our subsequent theory of differentiation.

8.1. Definition. We say $f$ is quasi-additive on $\mathfrak{F}$ if $f$ is a non-negative function whose domain $\mathfrak{W}$ is such a family of sets that

$$
\sum_{B \in \Theta} f(B) \leqq f(\beta)
$$

whenever $\sigma(\mathfrak{B}) \subset \beta \in \mathfrak{G}$ and $\mathcal{B}$ is a finite disjointed subfamily of $\mathfrak{F}$.

8.2. Definition. If $F$ is a blanket and $\alpha \subset S$ then we define $F \odot \alpha$ as the set $\alpha_{0}$ for which $x \in \alpha_{0}$ if and only if $x$ belongs to such an open set $T$ that

$$
F(x) \underset{S}{E}[S \subset T] \subset \underset{S}{E}[S \subset \alpha]
$$

8.3. Definition. We say $f$ is addivelous with respect to $F$ whenever $F$ is such a blanket with domain $A$ and spread $\mathfrak{F}$ that:

(i) $f$ is quasi-additive and finite valued on $\mathfrak{F}$;

(ii) if $\beta \in \mathfrak{F}$ and $\epsilon>0$, then there is a set $\alpha$ for which

$$
f(\alpha) \leqq f(\beta)+\epsilon, \quad \phi(\beta A-F \odot \alpha)=0 .
$$

8.4. REMARK. It should be noted that although 8.3 (ii) implicitly requires the membership of $\alpha$ in the domain of $f$ it does not require $\alpha \in \mathfrak{F}$.

In 8.3(i) we have specifically demanded that $f$ be finite valued on $\mathfrak{F}$. This is more a convenience than a necessity. As a matter of fact if we delete "and finite valued" from $8.3(i)$ and delete " $<\infty$ " from 8.10 below, the new Theorem 8.10 is valid in terms of the new Definition 8.3.

We make implicit use of the obvious fact that Definition 8.1 is equivalent to the statement obtained from it by replacing the word "finite" by "countable."

\subsection{TheOREM. If}

(i) $F$ is a strong blanket with domain $A$ and spread $\mathfrak{F}$,

(ii) $f$ and $\phi$ are addivelous with respect to $F$,

(iii) $B \in \mathfrak{F}$; 
then

$$
0 \leqq \operatorname{limal}_{P(x) \ni \beta \rightarrow x} \frac{f(\beta)}{\phi(\beta)}<\infty
$$

for almost all $x$ in $B A$.

Proof. For $x \in A$ we let

$$
D^{*}(x)=\varlimsup_{F(x) \ni \beta \rightarrow x} \frac{f(\beta)}{\phi(\beta)}, \quad D_{*}(x)=\underbrace{\operatorname{limal}}_{F(x) \ni \beta \rightarrow x} \frac{f(\beta)}{\phi(\beta)} .
$$

Whenever $g$ is addivelous with respect to $F$ we associate with $g$ that function $\bar{g}$ for which the domain of $\bar{g}$ is the set of all subsets of $S ; \bar{g}(S)$ is the infimum of numbers of the form

$$
\sum_{\beta \in \mathfrak{W}} g(\beta)
$$

where $\mathfrak{S}$ is such a countable subfamily of $\mathfrak{F}$ that $\mathfrak{S}$ covers almost all of $S$.

Bearing in mind that empty sums are 0 and that inf $0=\infty$, we readily check that $\bar{g}$ belongs to the (2.1) family $\mathfrak{I}$ whenever $g$ is addivelous with respect to $F$.

The remainder of the argument is divided into nine parts the first of which is obvious and the second of which, in a disguised form, concerns itself with the illegality of division by zero.

PART I. If $\gamma \subset S$ then $\phi(\gamma) \leqq \bar{\phi}(\gamma)$.

PART II. $0 \leqq D_{*}(x) \leqq D^{*}(x) \leqq \infty$ for almost all $x$ in $A$.

Proof. Let

$$
\gamma=A-\underset{x}{E}\left[0 \leqq D_{*}(x) \leqq D^{*}(x) \leqq \infty\right]
$$

and let $F^{\prime}$ be the function on $\gamma$ such that

$$
F^{\prime}(x)=F(x) \underset{\beta}{E}[\phi(\beta)=0] \quad \text { for } x \in \in^{\prime} \gamma
$$

Clearly $F^{\prime}$ is a subblanket of $F$ with domain $\gamma$. Hence by Definition 6.10 of a strong blanket we know $F^{\prime}$ is a heavy blanket, and that the spread of $F^{\prime}$ contains a countable disjointed family which covers almost all of $\boldsymbol{\gamma}$. Accordingly

$$
\phi(\gamma)=0 .
$$

PART III. If $0<\lambda<\infty, \gamma \subset A, g$ and $h$ are addivelous with respect to $F$, and if $F^{\prime}$ is such a subblanket of $F$ that

$$
\operatorname{limal}_{F^{\prime}(x) \ni S \rightarrow x} \frac{g(S)}{h(S)}<\lambda \quad \text { for } x \in \gamma
$$


then

$$
\bar{g}(\gamma) \leqq \lambda \bar{h}(\gamma) .
$$

We establish Part III by verifying Statements 1 and 2 below.

Statement 1. If $\beta \in \mathfrak{F}$, then

$$
\bar{g}(\beta \gamma) \leqq \lambda h(\beta) .
$$

Proof. Let $\epsilon>0$ and let $\alpha$ be a set for which (see 8.3)

$$
h(\alpha) \leqq h(\beta)+\epsilon, \quad \phi(\beta A-F \odot \alpha)=0 .
$$

Let $\alpha_{0}=F \odot \alpha$ and let $F^{\prime \prime}$ be the function on $\gamma \alpha_{0}$ such that

$$
F^{\prime \prime}(x)=F^{\prime}(x) \underset{S}{E}[S \subset \alpha, g(S)<\lambda h(S)] \quad \text { for } x \in \gamma \alpha_{0} .
$$

Definition 8.2 and the hypotheses of Part III assure us $F^{\prime \prime}$ is a subblanket of $F^{\prime}$. Consequently $F^{\prime \prime}$ is heavy.

Let therefore $\mathbb{S}$ be such a countable disjointed subfamily of the spread of $F^{\prime \prime}$ that $(S)$ covers almost all of $\gamma \alpha_{0}$. Since

$$
\begin{aligned}
\phi[\beta \gamma-\sigma(\Im))] & \left.\leqq \phi\left[\beta \gamma-\gamma \alpha_{0}\right]+\phi\left[\gamma \alpha_{0}-\sigma(\$)\right)\right] \\
& =\phi\left(\beta \gamma-\gamma \alpha_{0}\right)+0 \leqq \phi\left(\beta A-\alpha_{0}\right)=\phi(\beta A-F \odot \alpha)=0
\end{aligned}
$$

we infer $\&$ covers almost all of $\beta \gamma$. From the definition of $g$ and the quasiadditive nature of $h$ follows

$$
\bar{g}(\beta \gamma) \leqq \sum_{s \in \Theta} g(S) \leqq \lambda \sum_{S \in \Theta} h(S) \leqq \lambda h(\alpha) \leqq \lambda h(\beta)+\lambda \epsilon .
$$

STATEMENT 2. $\bar{g}(\gamma) \leqq \lambda \bar{h}(\gamma)$.

Proof. The conclusion being obvious in the event $h(\gamma)=\infty$ we assume $\bar{h}(\gamma)<\infty$.

Let $\epsilon>0$ and select from $\mathfrak{F}$ a countable subfamily $\mathfrak{W}$ which covers almost all of $\gamma$ in such a way that

$$
\sum_{\beta \in \mathfrak{S}} h(\beta) \leqq \bar{h}(\gamma)+\epsilon .
$$

In the light of Statement 1 and the membership of $g$ in the (2.1) family $\mathfrak{T}$, we see

$$
\begin{aligned}
\bar{g}(\gamma) & =\bar{g}(\gamma \sigma(\mathfrak{W}))=\bar{g}\left(\sum_{\beta \in \mathfrak{W}} \beta \gamma\right) \leqq \sum_{\beta \in \mathfrak{S}} \bar{g}(\beta \gamma) \\
& \leqq \lambda \sum_{\beta \in \mathfrak{S}} h(\beta) \leqq \lambda \bar{h}(\gamma)+\lambda \epsilon .
\end{aligned}
$$

PART IV. If $0<\xi<\infty, \gamma \subset A$, 


$$
D_{*}(x)<\xi
$$

then

$$
\bar{f}(\gamma) \leqq \xi \bar{\phi}(\gamma) .
$$

Proof. Let $F^{\prime}$ be such a subblanket of $F$ that

$$
\operatorname{limal}_{\boldsymbol{\gamma}^{\prime}(x) \ni \beta \rightarrow x} \frac{f(\beta)}{\phi(\beta)}<\xi \quad \text { for } x \in \gamma
$$

Let $g=f, h=\phi, \lambda=\xi$.

Apply Part III.

PART V. If $0<\eta<\infty, \gamma \subset A$,

$$
\eta<D^{*}(x)
$$

then

$$
\eta \bar{\phi}(\gamma) \leqq \bar{f}(\gamma) .
$$

Proof. Let $F^{\prime}$ be such a subblanket of $F$ that

$$
\operatorname{limal}_{\gamma^{\prime}(x) \ni \beta \rightarrow x} \frac{\phi(\beta)}{f(\beta)}<\frac{1}{\eta}
$$

Let $g=\phi, h=f, \lambda=1 / \eta$.

Apply Part III to obtain

$$
\bar{\phi}(\gamma) \leqq(1 / \eta) \bar{f}(\gamma), \quad \eta \bar{\phi}(\gamma) \leqq \bar{f}(\gamma) .
$$

PART VI. If $m$ and $n$ are positive integers and

$$
\gamma=A B \underset{x}{E}\left[D_{*}(x)<m / n<(m+1) / n<D^{*}(x)\right]
$$

then $\phi(\gamma)=0$.

Proof. Using Part V, Part IV, and the meaning of $\bar{\phi}(\gamma)$ we discover

$$
((m+1) / n) \bar{\phi}(\gamma) \leqq \bar{f}(\gamma) \leqq(m / n) \bar{\phi}(\gamma) \leqq(m / n) \phi(B)<\infty .
$$

Accordingly $\bar{\phi}(\gamma)=0$; and Part I insures

$$
\phi(\gamma)=0 .
$$

PART VII. $0 \leqq D_{*}(x)=D^{*}(x) \leqq \infty$ for almost all $x$ in $B A$.

Proof. Check that

$$
A B \underset{x}{E}\left[D_{*}(x)<D^{*}(x)\right]=\sum_{n=1}^{\infty} \sum_{m=1}^{\infty} A B \underset{x}{E}\left[D_{*}(x)<\frac{m}{n}<\frac{m+1}{n}<D^{*}(x)\right]
$$

and apply Parts VI and II.

PART VIII. $D^{*}(x)<\infty$ for almost all $x$ in $B A$. 
Proof. Let

$$
\gamma=A B \underset{x}{E}\left[D^{*}(x)=\infty\right]
$$

Part V tells us

$$
n \phi(\gamma) \leqq n \bar{\phi}(\gamma) \leqq \bar{f}(\gamma) \leqq f(B)<\infty \quad \text { for } 0<\eta<\infty .
$$

Hence $\phi(\gamma)=0$.

PART IX.

$$
0 \leqq \operatorname{limal}_{\boldsymbol{F}(x) \ni \beta \rightarrow x} \frac{f(\beta)}{\phi(\beta)}<\infty
$$

for almost all $x$ in $B A$.

Proof. Use Parts VII and VIII.

8.6. THEOREM. If

(i) F is a strong blanket with domain $A$ and spread $\mathfrak{F}$,

(ii) $f$ and $\phi$ are addivelous with respect to $F$, then

$$
0 \leqq \operatorname{limal}_{F(x) \ni \beta \rightarrow x} \frac{f(\beta)}{\phi(\beta)}<\infty
$$

for almost all $x$ in $A$.

Proof. We select from $\mathfrak{F}$ a countable disjointed subfamily $\&$ for which

$$
0=\phi[A-\sigma(\mathcal{S})]=\phi\left(A-\sum_{B \in \Theta} B A\right) .
$$

Application of 8.5 completes the proof.

8.7. Definition. For $\beta C S$ we denote the interior of $\beta$ by $\beta^{0}$.

Thus $\beta-\beta^{\circ}$ is contained in the boundary of $\beta$ whenever $\beta C S$.

8.8. Lemma. If $\beta \subset S, \alpha \subset S$, and $F$ is a blanket with domain $A$, then

$$
\phi(\beta A-F \odot \alpha) \leqq \phi\left(\beta-\alpha^{0}\right) .
$$

Proof. By taking $T=\alpha^{0}$ we easily infer from 8.2 that

$$
\begin{gathered}
x \in A \alpha^{0} \text { implies } x \in F \odot \alpha, \\
A \alpha^{0} \subset F \odot \alpha .
\end{gathered}
$$

Accordingly

$$
\beta A-F \odot \alpha \subset \beta A-A \alpha^{0}=\beta A-\alpha^{0} \subset \beta-\alpha^{0} .
$$

8.9. ThEOREM. If $F$ is a Borelish blanket and $f \in \mathfrak{i}$ then $f$ is addivelous with respect to $F$. 
Proof. Let $\mathfrak{F}$ be the spread of $F$. Evidently 8.3(i) is satisfied.

Suppose $\beta \in F$ and $\epsilon>0$. Since the bounded (6.1(ii)) set $\beta$ is contained in a bounded open set it is easy to infer from Theorem 3.7 of RM that a bounded open set $\alpha$ can be found for which

$$
\beta \subset \alpha=\alpha^{0}, \quad f(\alpha) \leqq f(\beta)+\epsilon, \quad \phi\left(\beta-\alpha^{0}\right)=0 .
$$

Consequently 8.8 forces the satisfaction of 8.3 (ii).

The proof is complete.

Our subsequent results on differentiation are more or less direct consequences of

8.10. Theorem. If $F$ is a strong blanket with domain $A$ and $f$ is addivelous with respect to $F$ then $\left.{ }^{10}\right)$,

$$
0 \leqq \operatorname{limal}_{F(x) \ni \beta \rightarrow x} \frac{f(\beta)}{\phi(\beta)}<\infty
$$

for almost all $x$ in $A$.

Proof. Apply 8.9 to $\phi$ and use 8.6.

From 8.10 and 8.9 follows

8.11. Theorem. If $f \in \mathfrak{i}$ and $F$ is a strong blanket with domain $A$ then

for almost all $x$ in $A$.

$$
0 \leqq \operatorname{limal}_{\boldsymbol{P}(x) \ni \beta \rightarrow x} \frac{f(\beta)}{\phi(\beta)}<\infty
$$

Our next theorem corresponds to and includes Lebesgue's theorem concerning the differentiability of a completely additive set function.

8.12. THEOREM. If $F$ is a strong blanket with domain $A, \mathfrak{B}$ is the family of all Borel subsets of $\mathcal{S}, f$ is completely additive and finite valued on $\mathfrak{B}$, then

$$
-\infty<\operatorname{limal}_{\mathbb{R}(x) \ni \beta \rightarrow x} \frac{f(\beta)}{\phi(\beta)}<\infty
$$

for almost all $x$ in $A$.

Proof. We ascertain $\left({ }^{11}\right)$ such functions $g_{1}$ and $g_{2}$ with domain $\mathfrak{B}$ that $g_{1}$

(10) If $A=S$ then Parts IV and $\mathrm{V}$ of the proof of 8.5 make it possible for us to see that

$$
\underset{x}{E}\left[\operatorname{limal}_{F(x) \ni \beta \rightarrow x} \frac{f(\beta)}{\phi(\beta)}>\lambda\right]
$$

is both $\bar{\phi}$ and $\phi$ measurable whenever $-\infty<\lambda<\infty$. This knowledge (which cannot be gained by the usual device) is indispensable to a study of the connection between our differentiation and the analogous integration.

(11) S. Saks, Theory of the integral, p. 11. 
and $g_{2}$ are finite valued, non-negative, and completely additive on $\mathfrak{B}$;

$$
f(\beta)=g_{1}(\beta)-g_{2}(\beta) \quad \text { for } \beta \in \mathfrak{B} \text {. }
$$

Now let $f_{1}$ and $f_{2}$ be the functions on the set of all subsets of $S$ for which

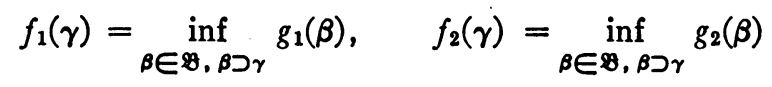

whenever $\gamma \subset \mathcal{S}$. Clearly $f_{1}$ and $f_{2}$ belong to $\mathfrak{i}$ and

$$
f(\beta)=f_{1}(\beta)-f_{2}(\beta)
$$

for $\beta \in \mathscr{B}$.

Reference to 8.11 completes the proof.

9. Elementary blankets. In this section we establish certain results which contribute to the differential theory of interval functions.

9.1. Definition. We say $F$ is an elementary blanket whenever $F$ is such a blanket with domain $A$ and spread $\mathfrak{F}$ that $\beta \in \mathfrak{F}$ implies $\phi(\beta A-F \odot \beta)=0$.

Many blankets met with in practice are elementary blankets. This is pointed out in

9.2. Theorem. If $F$ is such a blanket that $\phi\left(\beta-\beta^{0}\right)=0$ for $\beta$ in the spread of $F$, then $F$ is an elementary blanket.

Proof. Use 8.8.

9.3. Theorem. If

(i) $F$ is an elementary blanket with spread $\mathfrak{F}$,

(ii) $f$ is quasi-additive and finite valued on $\mathfrak{F}$, then $f$ is addivelous with respect to $F$.

Proof. Let $A$ be the domain of $F$. For $\beta \in F$ and $\epsilon>0$ we have

$$
f(\beta) \leqq f(\beta)+\epsilon, \quad \phi(\beta A-F \odot \beta)=0 .
$$

Recall 8.3.

An immediate consequence of 9.3 and 8.10 is

9.4. THEOREM. If

(i) $F$ is an elementary strong blanket with domain $A$ and spread $\mathfrak{F}$, then

(ii) $f$ is quasi-additive and finite valued on $\mathfrak{F}$,

$$
0 \leqq \operatorname{limal}_{P(x) \ni \beta \rightarrow x} \frac{f(\beta)}{\phi(\beta)}<\infty
$$

for almost all $x$ in $A$.

9.5. Definition. If $\mathfrak{S}$ is a family of nonvacuous sets and if $\mathbb{H}$ is such a subfamily of $\mathfrak{W}$ that no member of $\mathfrak{S}$ is contained in the product of two different members of $\mathbb{B}$ then we say $\mathbb{B}$ is $\mathfrak{W}$-disjointed. 
9.6. REMARK. If $\mathfrak{S}$ is a family of which 0 is a member then there are no $\mathfrak{W}$-disjointed families. On the other hand if 0 is not a member of $\mathfrak{E}$ then each disjointed subfamily of $\mathfrak{W}$ is $\mathfrak{W}$-disjointed. Moreover if $\mathfrak{W}$ is such a family of nonvacuous sets that $\beta_{1} \beta_{2} \in \mathfrak{S}$ whenever

$$
\beta_{1} \in \mathfrak{W}, \quad \beta_{2} \in \mathfrak{E}, \quad \beta_{1} \beta_{2} \neq 0
$$

then

$$
\text { (अ) is } \mathfrak{W} \text {-disjointed) }
$$

is equivalent to

(S) is a disjointed subfamily of $\mathfrak{E}$ ).

Finally, if $\mathfrak{E}$ is the family of all nondegenerate closed rectangles then

is equivalent to

(\$S is $\mathfrak{W}$-disjointed)

(S) $\subset \mathfrak{S})$ (no two different members of $(S)$ overlap).

9.7. Definition. If $f$ is a numerical valued function whose domain is a family $\mathfrak{E}$ of nonvacuous sets then $V_{f}$ is the function on $\mathfrak{S}$ such that $\beta \in \mathfrak{E}$ implies $V_{f}(\beta)$ is the supremum of numbers of the form

$$
\sum_{B \in @}|f(B)|
$$

where $\mathbb{B}$ is a finite $\mathfrak{W}$-disjointed family for which $\sigma(\mathbb{S}) \subset \beta$.

It is rather easy to verify

9.8. ThEOREM. If $f$ is a numerical valued function whose domain $\mathfrak{W}$ is a family of nonvacuous sets then not only is $V_{f}$ quasi-additive on $\mathfrak{S}$ but

$$
\sum_{B \in \Theta} V_{f}(B) \leqq V_{f}(\beta)
$$

whenever $\beta \in \mathfrak{S}$ and $\mathbb{S}$ is a finite $\mathfrak{D}$-disjointed family for which $\sigma(\mathbb{S}) \subset \beta$.

The next theorem may be of interest to some $\left({ }^{12}\right)$; it follows from 9.8 and 9.4.

9.9. ThEOREM. If

(i) $F$ is an elementary strong blanket with domain $A$ and spread $\mathfrak{F}$,

(ii) $V_{f}(\beta)<\infty$ for $\beta \in \mathfrak{F}$, then

$$
0 \leqq \varlimsup_{P(x) \ni \beta \rightarrow x} \frac{|f(\beta)|}{\phi(\beta)} \leqq \operatorname{limal}_{F(x) \ni \beta \rightarrow 0} \frac{V_{f}(\beta)}{\phi(\beta)}<\infty
$$

for almost all $x$ in $A$.

(12) S. Banach, Sur une classe de fonctions d'ensemble, Fund. Math. vol. 6 (1924) p. 176, Theorem 5. 
9.10 Definition. We say $\mathfrak{G}$ is a completion of $\mathfrak{F}$ whenever $\mathfrak{F}$ is such a subfamily of $\mathfrak{W}$ that: If $\sigma(\mathbb{H}) \subset \beta \in \mathfrak{W}$ and $\mathbb{B}$ is a finite disjointed subfamily of $\mathfrak{F}$, then $\mathfrak{B S}^{\mathfrak{S}}$ is contained in a finite $\mathfrak{S}$-disjointed family $\mathfrak{G}^{\prime}$ for which $\sigma\left(\mathbb{S}^{\prime}\right)=\beta$.

Thus if $\mathfrak{W}$ is the family of nondegenerate rectangles then $\mathfrak{W}$ is a completion of itself or any subfamily thereof. Note that the vacuous set is never a member of a completion of a family.

9.11. Definition. If $f$ is a numerical valued function whose domain $\mathfrak{S}$ is such a family that

$$
f(\beta) \leqq \sum_{B \in \mathcal{G}^{\prime}} f(B), \quad V_{f}(\beta)<\infty
$$

whenever $\beta \in \mathfrak{S}$ and $\mathcal{S}^{\prime}$ is a finite $\mathfrak{S}$-disjointed family for which $\beta=\sigma\left(\mathbb{S}^{\prime}\right)$, then we say $f$ is additively limited.

9.12. REMARK. If we should alter Definition 9.11 by replacing " $\leqq$ " by " $\geqq$ " then any function which is additively limited in the new sense is the negative of one which is additively limited in the old.

9.13. THEOREM. If

(i) $F$ is an elementary strong blanket with domain $A$ and spread $\mathfrak{F}$, then

(ii) $f$ is an additively limited function whose domain $\mathfrak{W}$ is a completion of $\mathfrak{F}$,

$$
-\infty<\operatorname{limal}_{F(x) \exists \beta \rightarrow x} \frac{f(\beta)}{\phi(\beta)}<\infty
$$

for almost all $x$ in $A$.

We divide the proof into two parts.

PART I. $V_{f}-f$ is quasi-additive on $\mathfrak{F}$.

Proof. Let $\sigma(\mathfrak{S )} \subset \beta \in \mathfrak{W}$ and suppose $\mathbb{B}$ is a finite subfamily of $\mathfrak{F}$. Bearing (ii) in mind, appropriately choosing $\mathcal{B S}^{\prime}$ in accordance with 9.10 , and noting the non-negativity of $V_{f}-f$, we use 9.8 and 9.11 to obtain

$$
\sum_{B \in \mathcal{G}^{\prime}}\left[V_{f}(B)-f(B)\right] \leqq \sum_{B \in \mathcal{G}^{\prime}}\left[V_{f}(B)-f(B)\right] \leqq V_{f}(\beta)-f(\beta) .
$$

PART II.

$$
-\infty<\operatorname{limal}_{F(x) \ni \beta \rightarrow x} \frac{f(\beta)}{\phi(\beta)}<\infty
$$

for almost all $x$ in $A$.

Proof. Since Part I, 9.8, and (ii) assure us $V_{f}-f$ and $V_{f}$ are quasi-additive and finite valued on $\mathfrak{F}$, we infer the desired conclusion from 9.4 and the relation 


$$
f=V_{f}-\left(V_{f}-f\right) \text {. }
$$

9.14. Remark. From Theorem 9.13 we not only deduce Lebesgue's theorem concerning the differentiability of an additive interval function, but, helped by 9.6 we also deduce a theorem of Banach $\left({ }^{18}\right)$ of which he made later use $\left({ }^{14}\right)$.

10. Nets.

10.1. Definition. We say $\mathfrak{E}$ is a net whenever $\mathfrak{E}$ is such a countable family of bounded nonvacuous Borel sets that:

$$
\mathfrak{S} \underset{\beta}{E}[\alpha \subset \beta]
$$

is a finite family for each $\alpha \in \mathfrak{W}$;

$$
\left(\beta_{1} \in \mathfrak{W}\right)\left(\beta_{2} \in \mathfrak{W}\right)\left(\beta_{1} \beta_{2} \neq 0\right)
$$

implies

$$
\left(\beta_{1} \subset \beta_{2}\right) \text { or }\left(\beta_{2} \subset \beta_{1}\right) \text {. }
$$

10.2. Definition. We say $\mathfrak{S}$ enmeshes $A$ whenever $\mathfrak{S}$ is such a net that corresponding to each positive number $\epsilon$ and each $x \in A$ there is a set $\beta \in \mathfrak{W}$ for which $x \in \beta$ and $\operatorname{diam} \beta<\epsilon$.

10.3. REMARK. If $\mathfrak{S}$ is a family of closed sets and $\mathfrak{E}$ enmeshes $A$ then $\mathfrak{E}$ covers $A$ in the sense of Vitali.

10.4. TEMPORARY Assumption. Throughout the remainder of this section we assume $\mathfrak{F}$ is a net enmeshing $A$ and assume $F$ is the function on $A$ such that $x \in A$ implies

$$
F(x)=\mathfrak{F} \underset{\beta}{E}[x \in \beta]
$$

10.5. TheOREM. $F$ is a strong blanket with domain $A$ and spread $\mathfrak{F}$.

Proof. Clearly $F$ is a blanket with domain $A$ and spread $\mathfrak{F}$.

In the next paragraph we show $F$ is a strong blanket.

Let $F^{\prime}$ be any subblanket of $F$ and denote the domain of $F^{\prime}$ by $A^{\prime}$ and the spread of $F^{\prime}$ by $\mathfrak{F}^{\prime}$. Evidently $\mathfrak{F}^{\prime}$ covers $A^{\prime}$. Let

$$
\mathfrak{B}^{\prime}=\mathfrak{F}^{\prime} \underset{\beta}{E}\left[\beta \subset \alpha \in \mathfrak{F}^{\prime} \text { implies } \beta=\alpha\right] \text {. }
$$

Using 10.1 we readily check that ${ }^{\prime \prime}{ }^{\prime}$ covers $A^{\prime}$ and that ${ }^{\prime \prime}{ }^{\prime}$ is a countable disjointed subfamily of $\mathfrak{F}^{\prime}$. Consequently $F^{\prime}$ is a heavy blanket.

10.6. REMARK. The theorems concerning differentiation with respect to a strong blanket are at our disposal.

(18) S. Banach, loc. cit. p. 177, Theorem 7.

(14) S. Banach, Sur les lignes rectifiable et les surfaces dont l'aire est finie, Fund. Math. vol. 7 (1925) p. 233. 
10.7. Lemma. If $\beta \in \Im$ then

$$
\beta A \subset F \odot \beta .
$$

Proof. Let $x \in \beta A$,

$$
\begin{aligned}
\mathfrak{Q} & =\mathfrak{F} \underset{\alpha}{E}[\beta \text { is a proper subset of } \alpha], \\
\eta & =\inf _{\alpha \in \mathfrak{S}} \operatorname{diam} \alpha .
\end{aligned}
$$

That $\eta>0$ follows from the finiteness of $\mathfrak{S}$ and the fact that every Borel set of diameter zero is closed (though it may have more than one point in it). Let $T$ be an open set for which

$$
x \in T, \operatorname{diam} T<\eta .
$$

Definition 10.1 now assures us of the relation

$$
F(x) \underset{\boldsymbol{B}}{E}[S \subset T] \subset \underset{\boldsymbol{B}}{E}[S \subset \beta]
$$

from which, according to 8.2 , follows

$$
x \in F \odot \beta \text {. }
$$

10.8. Theorem. $F$ is an elementary strong blanket.

Proof. Lemma 10.7 enables us to conclude

$$
\phi(\beta A-F \odot \beta)=0 \text { for } \beta \in \mathfrak{F} .
$$

Reference to 9.1 and 10.5 completes the proof.

10.9. ThEOREM. If $f$ is quasi-additive and finite valued on $\mathfrak{F}$ then

$$
0 \leqq \operatorname{limal}_{\boldsymbol{F}(x) \ni \beta \rightarrow x} \frac{f(\beta)}{\phi(\beta)}<\infty
$$

for almost all $x$ in $A$.

Proof. Use 10.8 and 9.4.

10.10. ThEOREM. If $\mathfrak{F}$ is a completion (9.10) of itself and $f$ is an additively (9.11) limited function whose domain is $\mathfrak{F}$, then

$$
-\infty<\operatorname{limal}_{\boldsymbol{P}(x) \ni \beta \rightarrow x} \frac{f(\beta)}{\phi(\beta)}<\infty
$$

for almost all $x$ in $A$.

Proof. Use 10.8 and 9.13.

10.11. REMARK. Since $\mathfrak{F}$ is a net it follows that a family is $\mathfrak{F}$-disjointed 
if and only if it is a disjointed subfamily of $\mathfrak{F}$.

It is obvious and well known that a given set is enmeshed by a net if and only if the given set is separable. Furthermore it is always possible to enmesh a compact set with a net which is a completion of itself. Thus corresponding to each separable set there is an elementary strong blanket whose domain is the separable set. Moreover, corresponding to each compact set there is an elementary strong blanket whose domain is the compact set and whose spread is a completion of itself.

10.12. TheOREM. $F$ is a regular Borelish blanket.

Proof. Let $\Delta$ be the function on $\mathfrak{F}$ such that $\beta \in \mathfrak{F}$ implies $\Delta(\beta)=3^{-n}$ where $n$ is the number of members of $\mathfrak{F}$ which contain $\beta$. Evidently $\Delta: \beta=\beta$ whenever $\beta \in \mathfrak{F}$. Consequently $F$ is regular.

10.13. REMARK. In the light of 3.7 of $R M$ it is now fairly easy to see that corresponding to each separable set there is a regular close blanket whose domain is the separable set.

11. Regular blankets.

11.1. Notation. Whenever $\sigma(\mathfrak{F}) \subset \mathcal{S}$ and $1 \leqq \lambda$ we define $\boldsymbol{\Xi}(\mathfrak{F}, \lambda)$ as the family $B$ for which $\beta \in B$ if and only if there is such a set $\alpha$ that

$$
\beta \subset \alpha \in \mathfrak{F}, \quad \phi(\alpha) \leqq \lambda \phi(\beta) .
$$

11.2. ThEOREM. If $f \in \mathfrak{i}$ and $F$ is a regular Borelish blanket with domain $A$ then

$$
0 \leqq \operatorname{limal}_{F(x) \ni \beta \rightarrow x} \frac{f(\beta)}{\phi(\beta)}<\infty
$$

for almost all $x$ in $A$.

Proof. Let $\Delta$ be such a non-negative function that

$$
\varlimsup_{P(x) \exists \beta \rightarrow x}\left(\Delta(\beta)+\frac{\phi(\Delta: \beta)}{\phi(\beta)}\right)<\infty
$$

for almost all $x$ in $A$. Abbreviate

$$
\underset{x}{E}\left[\varlimsup_{F(x) \ni \beta \rightarrow x}\left(\Delta(\beta)+\frac{\phi(\Delta: \beta)}{\phi(\beta)}\right)<n\right]
$$

by $A_{n}$. Note that

$$
A-\sum_{n=1}^{\infty} A_{n}
$$

has measure zero and that completion of the proof is tantamount to the verification of the 
Statement. If $n$ is a positive integer then

$$
0 \leqq \varlimsup_{F(x) \ni \beta \rightarrow x} \frac{f(\beta)}{\phi(\beta)}<\infty
$$

for almost all $x$ in $A_{n}$.

Proof. Let $F^{\prime}$ and $F^{*}$ be the functions on $A_{n}$ such that $x \in A_{n}$ implies

$$
\begin{aligned}
& F^{\prime}(x)=F(x) \underset{\beta}{E}\left[\Delta(\beta)+\frac{\phi(\Delta: \beta)}{\phi(\beta)}<n\right], \\
& F^{*}(x)=\Xi\left(F^{\prime}(x), 2\right) \underset{\beta}{E}[x \in \beta, \beta \text { is closed }] .
\end{aligned}
$$

The function $F^{\prime}$ is evidently a subblanket of $F$. In view of 3.7 of $\mathrm{RM}$ it is clear that $F^{*}$ is a close blanket. It is likewise clear that corresponding to each $\epsilon>0$ and each $\alpha$ in $F^{\prime}(x)$ there is a $\beta$ in $F^{*}(x)$ for which

$$
\beta \subset \alpha, \quad f(\alpha)-f(\beta)<\epsilon, \quad \phi(\alpha)-\phi(\beta)<\epsilon .
$$

Designating the spread of $F^{\prime}$ and the spread of $F^{*}$ respectively by $\mathfrak{F}^{\prime}$ and $\mathfrak{F}^{*}$, let us associate with each $\beta$ in $\mathfrak{F}^{*}$ a set $\bar{\beta}$ in $\mathfrak{F}^{\prime}$ in such a way that

$$
\beta \subset \bar{\beta}, \quad \phi(\bar{\beta}) \leqq 2 \phi(\beta) .
$$

In addition let us define $\Delta^{*}$ as the function on $\mathfrak{F}^{*}$ such that $\beta \in \mathfrak{F}^{*}$ implies

$$
\Delta^{*}(\beta)=\Delta(\bar{\beta}) .
$$

For $\beta \in \mathfrak{F}^{*}$ we first check that

$$
\Delta^{*}: \beta \subset \Delta: \bar{\beta}
$$

and then infer from (3) that

$$
\Delta^{*}(\beta)+\frac{\phi\left(\Delta^{*}: \beta\right)}{\phi(\beta)} \leqq \Delta(\bar{\beta})+\frac{2 \phi(\Delta: \bar{\beta})}{\phi(\bar{\beta})}<2 n .
$$

Since we now know $F^{*}$ is a regular close blanket we use $6.12,8.11,(2)$, and (1) in concluding

$$
0 \leqq \operatorname{limal}_{F^{*}(x) \ni \beta \rightarrow x} \frac{f(\beta)}{\phi(\beta)}=\operatorname{limal}_{F^{\prime}(x) \ni \beta \rightarrow x} \frac{f(\beta)}{\phi(\beta)}=\operatorname{limal}_{\boldsymbol{F}(x) \ni \beta \rightarrow x} \frac{f(\beta)}{\phi(\beta)}<\infty
$$

for almost all $x$ in $A_{n}$.

11.3. REMARK. Theorem 8.12 remains valid when "strong" is replaced by "regular Borelish."

An immediate consequence of $\mathbf{1 1 . 2}$ is 
11.4. THEOREM. If $f \in \mathfrak{i} \mathfrak{i}$ and $F$ is a diametrically regular Borelish blanket with domain $A$ then

$$
0 \leqq \operatorname{limal}_{F(x) \ni \beta \rightarrow x} \frac{f(\beta)}{\phi(\beta)}<\infty
$$

for almost all $x$ in $A$.

11.5. Definition. The family $\ddot{\mathfrak{B}}$ is defined by: $\psi \in \ddot{\mathfrak{B}}$ if and only if $\psi$ is such a member of $\mathfrak{i}$ that corresponding to each $S \subset S$ there is a Borel set $C$ for which

$$
S \subset C, \quad \psi(S)=\psi(C) .
$$

11.6. THEOREM. If $f$ and $\phi$ belong to $\ddot{\mathfrak{B}}$ and $F$ is a diametrically regular blanket with domain $A$ then

$$
0 \leqq \operatorname{limal}_{F(x) \ni \beta \rightarrow x} \frac{f(\beta)}{\phi(\beta)}<\infty
$$

for almost all $x$ in $A$.

Proof. Let $F^{*}$ be the function on $A$ such that $x \in A$ implies $F^{*}(x)$ is the family defined by $\beta \in F^{*}(x)$ if and only if

$$
\beta \text { is a Borel set, } \quad \alpha \subset \beta \subset \text { closure } \alpha
$$

for some $\alpha$ in $F(x)$.

By defining $\Delta$ as the function on $E_{S}[S \subset S]$ such that

$$
\Delta(S)=(\operatorname{diam} S)^{2} \text { whenever } S \subset \mathcal{S},
$$

we see that $F^{*}$ is a regular Borelish blanket and employ 11.2 in checking

$$
0 \leqq \operatorname{limal}_{F^{*}(x) \ni \beta \rightarrow x} \frac{f(\beta)}{\phi(\beta)}=\operatorname{limal}_{F(x) \ni \beta \rightarrow x} \frac{f(\beta)}{\phi(\beta)}<\infty
$$

for almost all $x$ in $A$.

11.7. REMARK. If we delete the word "diametrically" from 11.6 the resulting statement is false.

11.8. REMARK. In view of Theorem 10.12 it is natural to ask: Is there always a diametrically regular blanket with domain $A$ whenever $A$ is a separable set? The answer is no, as may be seen by taking $A=S=E_{t}[-\infty<t<\infty]$, $\phi=$ linear Lebesque measure, and defining $\rho$ in some manner sufficiently different from the usual.

However certain positive results are obtainable.

We denote the closed sphere

$$
\underset{y}{E}[\rho(x, y) \leqq r]
$$


by $\mathbb{E}_{x}^{r}$. We suppose $F$ is the function on $A \subset \mathcal{S}$ such that $x \in A$ implies

$$
F(x)=\sum_{0<r<\infty}\left\{\mathfrak{S}_{x}^{r}\right\} .
$$

Thus for each $x$ in $A, F(x)$ is the family of all closed spheres with center $x$ and positive finite radius. It is easy to verify: if

$$
\lim _{n \rightarrow \infty} \varlimsup_{r \rightarrow 0} \frac{\phi\left(\mathcal{S}_{x}^{r}\right)}{r^{n}}=\infty
$$

for almost all $x$ in $A$, then there is a diametrically regular subblanket of $F$ with domain $A$. More specifically if $A=S=m$-space and $\rho$ is the usual metric then, no matter what $\phi \in \mathfrak{i}$ we have chosen, it is true that there is a diametrically regular subblanket of $F$ with domain $A$; of course. if $\phi$ is an $m$-dimensional Lebesgue measure then $F$ itself is a diametrically regular blanket.

12. Comparative regularity. We use notation 11.1 in

12.1. Definition. If $F$ is a blanket with domain $A$ and if there is such a strong blanket $F_{0}$ and such a positive finite valued function $\Lambda$ that

$$
F(x) \subset \Xi\left(F_{0}(x), \Lambda(x)\right)
$$

for almost all $x$ in $A$, then we say $F$ is comparatively regular.

From 6.12 we obtain

12.2. THEOR EM. Every regular close blanket is comparatively regular. blanket.

12.3. LemMa. If $F$ is a comparatively regular close blanket then $F$ is a heavy

Proof. Let $A$ and $\mathfrak{F}$ respectively be the domain and spread of $F$. Let $F_{0}$ be the strong blanket whose existence is assured by 12.1. Let $\Omega$ be such a countable family of bounded sets that:

$$
\phi(A-\sigma(\Re))=0 ;
$$

corresponding to each $S \in \Omega$ there is an integer $n$ for which

whenever $x \in S$.

$$
F(x) \subset \Xi\left(F_{0}(x), n\right)
$$

The lemma now follows from Theorem 5.2 and the

Statement. If $S \in \Re$ then $\mathfrak{F}$ has the Vitali property with respect to $S$.

Proof. Let $\mathfrak{S}$ be a finite disjointed subfamily of $\mathfrak{F}$. Let $n$ be a positive integer such that

$$
F(x) \subset \Xi\left(F_{0}(x), n\right)
$$

whenever $x \in S$. Let $T$ be a bounded open set containing $S+\sigma(\mathfrak{E})$. Select such 
a sequence $G$ of finite disjointed families that:

$$
\mathfrak{S} \subset G, \subset G_{\nu+1} \subset \mathfrak{F}, \quad \sigma\left(G_{\nu}\right) \subset T
$$

for each positive integer $\nu$;

$$
\phi\left[\sigma\left(G_{\nu+1}\right)\right]+1 / \nu \geqq \phi[\sigma(\xi)]
$$

whenever $\nu$ is a positive integer and $\notin$ is a finite disjointed family for which

$$
G, \subset \mathfrak{F} \subset \text { F, } \quad \sigma(\mathfrak{F}) \subset T .
$$

Defining

$$
\mathbb{S}=\sum_{p=1}^{\infty} G_{\nu},
$$

we see that $\mathbb{B}$ is a countable disjointed family for which $\mathfrak{S} \subset \& \subset \mathfrak{F}$. Moreover from the definition of $G$, the positivity of $1 / n$, and the strength of $F_{0}$, we conclude the falsity of

$$
\phi[S-\sigma(\mathfrak{S})]>0 .
$$

The proof is complete.

We notice that any subblanket of a comparatively regular blanket is also comparatively regular. Consequently 12.3 yields

12.4. TheOREM. If $F$ is a comparatively regular close blanket then $F$ is a strong blanket.

12.5. THEOREM. If $f \in \mathfrak{i}$ and $F$ is a comparatively regular Borelish blanket with domain $A$ then

for almost all $x$ in $A$.

$$
0 \leqq \operatorname{limal}_{P(x) \ni \beta \rightarrow x} \frac{f(\beta)}{\phi(\beta)}<\infty
$$

Proof. Let $F^{*}$ be the function on $A$ such that $x \in A$ implies

$$
F^{*}(x)=\Xi(F(x), 2) \underset{\beta}{E}[\beta \text { is closed }] \text {. }
$$

In the light of Theorem 3.7 of $\mathrm{RM}$ we see $F^{*}$ is a comparatively regular close blanket. Using 12.4, 8.11, and re-using 3.7 of $\mathrm{RM}$ we discover

$$
0 \leqq \operatorname{limal}_{F^{*}(x) \ni \beta \rightarrow x} \frac{f(\beta)}{\phi(\beta)}=\operatorname{limal}_{F(x) \ni \beta \rightarrow x} \frac{f(\beta)}{\phi(\beta)}<\infty
$$

for almost all $x$ in $A$.

12.6. REMARK. Theorem 8.12 remains valid when "strong" is replaced by "comparatively regular Borelish." 
12.7. ThEOREM. If $f$ and $\phi$ belong (11.5) to $\ddot{\mathfrak{B}}$ and $F$ is a comparatively regular blanket with domain $A$ then

$$
0 \leqq \operatorname{limal}_{P(x) \ni \beta \rightarrow x} \frac{f(\beta)}{\phi(\beta)}<\infty
$$

for almost all $x$ in $A$.

Proof. With the help of 11.5 and 12.1 secure such a comparatively regular Borelish blanket $F^{*}$ that $\alpha \in F(x)$ implies the existence of a $\beta$ in $F^{*}(x)$ for which

$$
\alpha \subset \beta \subset \text { closure } \alpha, \quad \phi(\beta)=\phi(\alpha), \quad f(\beta)=f(\alpha) .
$$

From 12.5 it now follows that

$$
0 \leqq \operatorname{limal}_{\boldsymbol{F}^{*}(x) \ni \beta \rightarrow x} \frac{f(\beta)}{\phi(\beta)}=\operatorname{limal}_{\boldsymbol{P}(x) \ni \beta \rightarrow x} \frac{f(\beta)}{\phi(\beta)}<\infty
$$

for almost all $x$ in $A$.

13. Further uses for covering theorems. In this section we illustrate the applicability of $\$ \$ 3$ through 6 to certain problems akin to some which arise in connection with Carathéodory linear measure and Hausdorff dimensions $\left({ }^{15}\right)$.

Let $\xi$ be such a positive nondecreasing function on $E_{t}[0<t<1]$ that

$$
\varlimsup_{t \rightarrow 0+} \frac{\xi(5 t)}{\xi(t)}<\infty .
$$

We note that condition (1) is satisfied if $0<\alpha<\infty$ and

$$
\xi(t)=t^{\alpha} \text { for } 0 \leqq t<\infty .
$$

For $0<r$ we define $\xi_{r}$ as the function on $E_{S}[S \subset S]$ such that $S \subset S$ implies $\xi_{r}(S)$ is the infimum of numbers of the form

$$
\sum_{\beta \in \mathfrak{Q}} \xi(\operatorname{diam} \beta)
$$

where $\mathfrak{W}$ is such a countable family of nonvacuous sets that

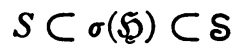

and

$$
0<\operatorname{diam} \beta<r \text { whenever } \beta \in \mathfrak{W} \text {. }
$$

Let $\psi$ be the function on $E_{S}[S \subset S]$ such that $S \subset S$ implies

$$
\psi(S)=\lim _{r \rightarrow 0+} \xi_{r}(S) \text {. }
$$

(15) F. Hausdorff, Dimension und ausseres Mass, Math. Ann. vol. 79 (1919) pp. 157-179. 
It is easy to check that $\psi \in \mathfrak{U}$. Further scrutiny reveals that every subset of $S$ is contained in an $F_{\sigma \delta}$ with the same measure.

Next we fix a set $A$ for which $\psi(A)<\infty$ and let $\Psi$ be the function on $E_{S}[S \subset S]$ such that

$$
\Psi(S)=\psi(A S) \text { for } S \subset \mathcal{S} .
$$

Not only does $\Psi$ belong to $\ddot{\mathfrak{i}}$ but (11.5) to $\ddot{\mathfrak{B}}$ as well. In fact from 3.7 of $\mathrm{RM}$ we deduce

Statement I. Corresponding to each $\epsilon>0$ and each $\beta C S$ there is an open set $\alpha$ for which

$$
\beta \subset \alpha, \quad \Psi(\alpha) \leqq \Psi(\beta)+\epsilon .
$$

Next we let $F$ be the function on $A$ such that $x \in A$ implies

$$
F(x)=\underset{\beta}{E}[\beta \text { is closed, } x \in \beta, 0<\operatorname{diam} \beta<\infty]
$$

and observe that $F$ is a blanket with domain $A$. By making use of 3.5 , (1), and the definitions of $\psi$ and $\Psi$ we readily check

$$
\varlimsup_{\boldsymbol{r}(\boldsymbol{x}) \ni \beta \rightarrow x} \frac{\Psi(\beta)}{\xi(\operatorname{diam} \beta)}<\infty
$$

for $\psi$ almost all $x$ in $A$.

We now identify our function $\phi$ with the function $\Psi$ under discussion. From (2) and (1) we obtain

Statement II. If $F^{\prime}$ is a subblanket of $F$, the domain of $F^{\prime}$ is $A_{0}$, and if

$$
\varliminf_{F^{\prime}(x) \ni \beta \rightarrow x} \frac{\Psi(\beta)}{\xi(\operatorname{diam} \beta)}>0
$$

for $\psi$ almost all $x$ in $A_{0}$, then $F^{\prime}$ is a diametrically regular close blanket.

In view of 6.12 we see that Statement II includes a theorem $\left({ }^{16}\right)$ of Randolph.

From Statement I and the definitions involved we infer by direct methods that

$$
\varlimsup_{F(x) \ni \beta \rightarrow x} \frac{\Psi(\beta)}{\xi(\operatorname{diam} \beta)} \leqq 1
$$

for $\psi$ almost all $x$ in $A$. On the other hand use of Statement I, Stätement II, and 6.12 yields

$$
\varlimsup_{F(x) \ni \beta \rightarrow x} \frac{\Psi(\beta)}{\xi(\operatorname{diam} \beta)} \geqq 1
$$

(16) J. F. Randolph, loc. cit. footnote 1, p. 299, Theorem 1. 
for $\psi$ almost all $x$ in $A$.

We thus have

Statement III.

$$
\varlimsup_{F(x) \ni \beta \rightarrow x} \frac{\psi(A \beta)}{\xi(\operatorname{diam} \beta)}=1
$$

for $\psi$ almost all $x$ in $A$.

Moreover with the aid of our 3.5, (1), Theorem 3.13 of RM, and the technique used in proving Theorem 5.12 of RM we discover

Statement IV. If $A$ is $\psi$ measurable, $C \subset S-A$, and $F^{*}$ is such a blanket with domain $C$ that

$$
F^{*}(x) \subset \underset{\beta}{E}[\beta \text { is closed, } x \in \beta, 0<\operatorname{diam} \beta<\infty]
$$

whenever $x \in C$, then

$$
\varlimsup_{F(x) \ni \beta \rightarrow x} \frac{\psi(A \beta)}{\xi(\operatorname{diam} \beta)}=0
$$

for $\psi$ almost all $x$ in $C$.

Let us now be more specific. Suppose $S=E_{t}[-\infty<t<\infty]$ and $\rho$ is the usual metric. Suppose $0<\alpha \leqq 1$,

$$
\xi(t)=t^{\alpha} \text { for } 0<t<\infty .
$$

Let $f$ be the function on $E_{t}[-\infty \rightarrow t<\infty]$ such that

$$
f(x)=\psi(A \underset{t}{E}[-\infty<t \leqq x]) \text { for }-\infty<x<\infty .
$$

From Statement III we conclude

STATEMENT V.

$$
\varlimsup_{h \rightarrow 0} \frac{|f(x+h)-f(x)|}{|h|^{\alpha}}=1
$$

for $\psi$ almost all $x$ in $A$.

The University of California,

Berkeley, Calif. 